\title{
Major Antigen Genotypes and Molecular Epidemiological Analysis of Bordetella Pertussis Isolated in Shenzhen
}

\section{Shuang Wu}

shenzhen CDC: Shenzhen Center for Disease Control and Prevention

\section{Qinghua Hu}

shenzhen CDC: Shenzhen Center for Disease Control and Prevention

Chao Yang

shenzhen CDC: Shenzhen Center for Disease Control and Prevention

Haijian Zhou

ICDC: National Institute for Communicable Disease Control and Prevention

\section{Hongyu Chen}

Shenzhen Children's Hospital

\section{Yanwei Zhang}

shenzhen CDC: Shenzhen Center for Disease Control and Prevention

\section{Min Jiang}

shenzhen CDC: Shenzhen Center for Disease Control and Prevention

\section{Yuxiang He}

shenzhen CDC: Shenzhen Center for Disease Control and Prevention

Xiaolu Shi ( $\square$ shixiaolu831@163.com )

shenzhen CDC: Shenzhen Center for Disease Control and Prevention

\section{Research Article}

Keywords: Bordetella pertussis, genotype, pertussis vaccine, molecular epidemiology

Posted Date: March 5th, 2021

DOl: https://doi.org/10.21203/rs.3.rs-268844/v1

License: (c) (1) This work is licensed under a Creative Commons Attribution 4.0 International License. Read Full License

Version of Record: A version of this preprint was published at Annals of Clinical Microbiology and Antimicrobials on August 18th, 2021. See the published version at https://doi.org/10.1186/s12941-021- 
00458-3.

Page 2/18 


\section{Abstract}

\section{Background}

Although the global epidemic of pertussis has been controlled through the expanded Programme on Immunization (EPI), the incidence of pertussis has increased significantly in recent years, with a "resurgence" of pertussis occurring in developed countries with high immunization coverage. The incidence of pertussis in Shenzhen, was about 2.02/100,000, far exceeding that of the whole province and the whole country (both $<1 / 100,000$ ). At the same time, more and more studies have shown that there is antigenic drift in Bortella pertussis, which may be associated with the increased incidence. 50 strains of Bordetella pertussis isolated from 387 suspected cases were collected in Shenzhen in 2018 for genotype distributions and molecular epidemiological characteristics analysis.

Methods

There were 387 suspected cases of pertussis enrolled at surveillance sites in Shenzhen from June to August 2018. Nasopharyngeal swabs of suspicious cases were collected for separation and culture, and the positive strains were identified by real-time PCR. The immunization histories of patients were analyzed to investigate the relationship between pertussis vaccination and infection. The major antigen genes of the isolated positive strains, including $p t x A, p t x C$, ptxP, prn, fim2, and fim3, were analyzed by second-generation sequencing. The homology and phylogenetic analysis of these genes was performed using the public genome sequence downloaded from GenBank.

Results

50 strains of Bordetella pertussis were successfully isolated from nasopharyngeal swabs of 387 suspected cases, with a positive rate of $12.9 \%$, including 28 males and 22 females, accounting for $56.0 \%$ and $44.0 \%$ respectively. It is worth noting that 38 were under one-year-old among the positive patients, accounting for $76.0 \%$. Among the cases with a history of vaccination, $71.4 \%$ of positive patients did not complete the basic vaccination process of the DTaP at the time of onset. Three major antigen genotypes different from CS and Tohama I vaccine strains were identified, and they had distant genetic relationships and $62.0 \%$ of which was prn2/ptxC2/ptxP3/ptxA1/fim3-1/fim2-1.

\section{Conclusions}

The positive rate of cases under one-year-old was significantly higher than that of other age groups and should be monitored. The major antigenic genes of the Bordetella pertussis strains isolated in Shenzhen were different from those of common vaccine strains. This study explained the resurgence of whooping cough from certain angles, including immunization strategy, vaccination time and genome variation of strains, which is beneficial to prevent pertussis infections.

\section{Background}


Pertussis is an acute respiratory infectious disease caused by the gram-negative bacterium Bordetella pertussis[1]. The typical symptoms of pertussis include paroxysmal coughing with an inspiratory whoop, post-tussive vomiting, cyanosis, and persistent coryzal symptoms[2]. Pertussis is mainly transmitted by droplets, and infection sources are mainly early patients and carriers. People are generally susceptible to pertussis, especially infants under one year old[3]. The whole-cell pertussis vaccine (WPV) was invented in 1914 , then combined with tetanus and diphtheria toxoids in the 1940 s to become widely used diphtheria-tetanus-pertussis $\mathbb{D} \mathrm{DTP} \otimes$ vaccine, which is about $80 \%$ effective in preventing severe illness and death from pertussis. Since then, a successive decline in the incidence of the disease has been observed[4]. However, due to the high frequency of adverse events following WPV immunization, many parents refused to vaccinate their children and lawsuits against the vaccine manufacturers forced many of them to stop producing the vaccine $[5,6]$. Therefore, the pertussis vaccine currently used in the world is mainly Acellular Pertussis Vaccine (APV). Studies have confirmed that compared with WPV, APV can significantly reduce the adverse effects on the body[1]. But in the past decade, pertussis incidence has risen again, and the so-called whooping-cough epidemics have reappeared[7, 8]. According to the World Health Organization (WHO), as many as 195,000 children worldwide died of pertussis and its complications in 2008 , with $90 \%$ of cases occurring in less developed and developing countries[9]. Even in developed countries or countries with high pertussis vaccination rates, the incidence of pertussis has been on the rise in recent years[10]. In 2012, there was an outbreak of pertussis in Washington and other states in the United States, with the highest reported incidence $(37.5 / 100,000)$ since 1942. It is worth noting that $43 \%$ of patients had at least 4 doses of immunological history of acellular DPT vaccine (DTaP)[11]. APV vaccine in China mainly contains purified pertussis toxoid (PT) and filamentous hemagglutinin (FHA), and some imported vaccines have additional pertussis adhesion protein (Pertactin, PRN) or fimbriae (FIM) antigens $[12,13]$. The production process of APV can be divided into two types: 1 . Co-purification of chemically detoxified PT and FHA; 2. Separate purification of PT, FHA and 1 3 types of pertactin $\triangle P R N)$. Historically, countries around the world have used modified intra-cerebral challenge assay (MICA), the only effective method that has obtained clinically verified protection on the evaluation of pertussis vaccine, to evaluate the effectiveness of WPV and its stock solution.

In recent years, the incidence of pertussis in adolescent and adult has been increasing year by year, but the clinical symptoms are not typical and the epidemiological characteristics have also changed[14-16]. Vaccines are the main way to prevent pertussis, which have been widely used for immunization in since 1960s[17]. Shenzhen adopted whole-cell vaccines before 2008, and gradually replaced it with acellular vaccines from 2008 to 2010. Since 2010, acellular vaccines have been used throughout the city. According to the national planned immunization program, the age of vaccination is $3,4,5$, and 18 months after birth. The vaccination rate has kept above $99 \%$ in recent years. However, according to the statistics of the China Information System for Disease Control and Prevention, the number of reported cases of pertussis in Shenzhen is rising sharply. Studies have shown that pathogens have undergone adaptive changes under the pressure of immune protection pressure induced by the body after vaccination. The PT, PRN, FIM and other genes encoding vaccine-related antigens of current popular 
strains are different from vaccine strains $[18,19]$. Thereby, they could evade the body's immune protection response.

To further explain the possible impact of the genetic difference on the immune effects, and to better understand the pathogenic characteristics, evolutionary characteristics, and molecular epidemiological rules of isolated Bordetella pertussis in China, we sequenced 50 strains of Bordetella pertussis isolated from Shenzhen in 2018 to analyze their population structure and sequence characteristics of major antigen gene.

\section{Material And Methods}

Research object. 387 suspected pertussis cases from the outpatient department of Shenzhen Children's Hospital from June to August 2018.

\section{Main reagents and instruments}

Main reagents. Carbon agar medium (Oxoid company, Canada), Pertussis Bacteria Phase I Standard Serum (REMEL company, Lenexa City, KS, USA) and Whole-genome DNA extraction kit (Qiagen Company, Shanghai, China).

Main instruments. Fluorescence PCR instrument (ABI Company, Oyster Bay, NY, USA) and NonoDrop1000 Ultra Micro Spectrophotometer (Thermo Fisher Company, Fair Lawn, NJ, USA).

Culture. The samples were cultured on the carbon agar plate at $37^{\circ} \mathrm{C}$ for 3 days. Round, moist, protruding, transparent or translucent suspected colonies were taken for pure culture and then identified by biochemical reaction and agglutination tests. Simultaneously, DNA was extracted from bacterial precipitation and the pertussis-specific genes IS481 and $p t x A$ were detected by Real-time PCR method.

Genome sequencing and de novo assembly. The genomic DNA of Bordetella pertussis was extracted using the whole-genome DNA extraction kits according to the manufacturer's instructions. The library was constructed using the Illumina HiSeq 4000 system (Illumina, San Diego, CA, USA) at the Beijing Genomics Institute (Shenzhen, China). DNA sample preparation kit (Illumina, San Diego, CA, USA), and whole gene sequencing and preliminary evaluation filtering was conducted using the Illuminas HiSeq X-Ten platform (BGI, Shenzhen, China). The original data was further filtered by SOAPnuke to obtain valid data via quality control. De novo assembly of the genome was performed by SPAdes gene assembly software (V3.9.1).

Sequence analysis of major antigen genes. The sequence numbers of the reference genes are as follows:

ptxA gene (vaccine CS strain ptxA2 type: WP_010931648.1, Tohama I strain ptxA2 type: NP_882282.1, B592 strain ptxA2 type: AJ245367.1, 287 strain ptxA1 type: AJ006155.1, B6 strain ptxA4 type: AJ506336.1); 
ptxC gene (Tohama I strain ptxC1 type:NP_882286.1, 3779 strain $p t x C 1$ type: AAA22985.1, NK strain ptxC2 type: AJ420987.1);

ptxP gene (Tohama I strain ptxP1 type: FN252323.1, B2983 strain ptxP3 type: FN252324.1);

prn gene(vaccine CS strain:WP_010930159 .1, Tohama I strain: NP_879839.1, B391 strain prn1 type: AJ011091.1, B345 strain prn2 type: AJ011092.1, B343 strain prn3 type: AJ011093.1)『

fim2 gene (vaccine CS strain fim2-1 type: WP_010930199.1, Tohama I strain: NP_879898.1, NK strain fim2-2 type: AJ420988.1)凹

fim3 gene (vaccine CS strain: WP_010930436.1, Tohama I strain fim3-1 type : NP_880302.1, fim3-2 type: AY464179.1, fim3-3 type:AY464180.1, fim3-4 type: AY464181.1). The homologous sequences of virulence factors in the genome sequence of Shenzhen strains were extracted using Blastn, and the sequence alignment and phylogenetic analysis on the homologous genes of ptxA, ptxC, ptxP, prn, fim2 and fim3 sequences was performed using Mega 7.0 software.

\section{Single nucleotide polymorphism (SNP) extraction and phylogenetic typing.}

842 public genome sequences were downloaded from GenBank (https://www.ncbi.nlm.nih.gov/genome/browse\#!/prokaryotes/1008/, as of September 2020). Snippy software (https://github.com/tseemann/snippy/) was used to detect the core genome SNP sites of the public sequence and the Shenzhen strain sequence, and the genome sequence of Tohama I strain (GenBank number: BX470248.1) was used as a reference. TRF software (https://tandem.bu.edu/trf/trf.html) and BLASTn were used to identify the recombination region of the reference genome and to remove the SNP sites located in the repeating region. Based on non-repetitive regions of SNP sites, the maximum likelihood phylogenetic tree was built using IQ-Tree software.

\section{Results}

\section{Laboratory test results and epidemiological characteristics analysis.}

50 strains of Bordetella pertussis were obtained from nasopharyngeal swabs of 387 children with bedside inoculation, with a positive rate of $12.9 \%$ after bedside inoculation. The epidemiological characteristics of patients were shown in Table 1. For the vaccination status of children with pertussis, 8 children did not provide detailed vaccination information. Of the remaining 42 children, 11 cases did not vaccinate the pertussis vaccine (26.2\%), 19 cases did not complete the basic immunization process (45.2\%), 5 cases completed the basic immunization process (11.9\%), and 7 have completed the booster immunization process $(16.7 \%)$. Taken together, $71.4 \%$ of children did not complete the basic pertussis vaccination program at the time of onset.

Major antigen gene analysis. Among the 6 antigen genes isolated, 2 had 100\% homology with the vaccine strains CS and Tohamal, namely fim2-1 and fim3-1 respectively. The other four genes have 
changed their nucleotide sequence, resulting in a change in antigen type. Among them, all ptxA genes had non-synonymous mutations. According to evolutionary analysis, it can be seen that, the isolated strain was different from the vaccine strain ptxA2 and $100 \%$ homologous to the 287 strain ptxA1 type (Figure 1). Similarly, non-synonymous mutations occurred in the $p t x P$ gene. Except 19 strains had the same gene sequence as the vaccine strain, the remaining 31 strains became ptxP3 (Figure 2). The synonymous mutation occurred in the $p t x C$ gene, and 31 isolates became $p t x C 2$ (Figure 3). The prn genes of 50 isolates included 3 types , $18 \mathrm{prn} 1,31 \mathrm{prn} 2$ and one prn3. The major antigen genotypes of the 50 isolates can be divided into 3 categories, including 18 strains (36\%) of prn 1/ptxC1/ptxP1/ptxA1/fim3-1/fim2-1,31 strains (62\%) of prn2/ptxC2/ptxP3/ptxA1/ fim3-1/fim2-1, and one strain of (2\%) prn3/ptxC1/ptxP1/ptxA1/fim3-1/fim2-1 (Table 2).

\section{Genome evolution analysis.}

Genetic analysis was performed on 50 isolates and 842 pertussis strains with known sequences worldwide, and the results were shown in Figure 5. The Shenzhen isolates in this study were distributed in three evolutionary branches (phylogenetic group, PG). Genotype of prn2/ptxA1/ptxP3/ptxC2/fim3-1/ fim2-1 is concentrated in PG1, genotype of prn3/ptxA1/ptxP1/ptxC1/fim3-1/fim2-1 is located in PG2, and genotype prn 1/ptxA1/ptxP1/ptxC1/fim3-1/fim2-1 are concentrated in PG3. The isolates from PG3 are closely related to the epidemic strains in northern China in recent years, but far from foreign strains. All isolates were genetically distant from vaccine strains CS and Tohama I.

\section{Discussion}

Among the genus of Bordetella, Bordetella pertussis is the only bacteria that can produce PT, which is the main virulence factor of Bordetella pertussis and consists of five subunits. Among them, subunit $A$ is the main functional subunit, which has immunoprotective properties and a variety of enzyme activities, and is expressed by ptxA gene[20]. The region between amino acid 65 and 233 is the conserved sequence of pertussis toxin, causing the host cell immune response and is the antigen recognition site of $T$ cells. According to the difference of the 68th, 228th, and 232nd amino acids of the Subunit A, the ptxA gene is divided into four subtypes, 1, 2, 3 and 4[21]. The ptxA gene subtypes of pertussis vaccine strains and strains isolated before or early in vaccination are reported to be ptxA2 or ptxA4 subtypes in many countries in the world. The reference strains Tohama I and CS in this study belong to ptxA2 subtype strain, while all of our 50 isolates had non-synonymous mutations ( $G$ to $A$ ), that is, from the original leucine (I) to methionine (M), and belong to the ptxA1 subtype strain. The protective effect of the vaccine produced by the vaccine containing the $p t x A 2$ vaccine strain is weak against the strain containing the ptxA1 gene[22].

The promoter of upstream pertussis toxin gene ( $p t x P)$ region of the PT gene of Bordetella pertussis is composed of approximately 170 bases, compared with Bordetella parapertussis and Bordetella bronchitis, which is species-specific characteristics[23]. This region contains binding sites for RNA polymerase and 6 binding sites of bvg A regulatory protein dimers, which can promote the transcriptional 
expression of PT by interacting with the regulatory protein bvg A. In recent years, researchers have discovered that Bordetella pertussisPtxP presents a certain polymorphism, and according to the mutation bases at specific positions, Bordetella pertussisPtxP is divided into 11 subtypes. Dutch researchers have confirmed that strains containing ptxP3 can highly express PT and have gradually become the dominant epidemic strains in the Netherlands. It is speculated that the "pertussis recurrence" may be related to this. Therefore, the ptxP gene of Bordetella pertussis has attracted more and more attention and has been used as a molecular genetic marker for the evolution of Bordetella pertussis[10]. PtxP3 accounted for $62 \%$ of the 50 pertussis strains isolated in this study, which is consistent with the international trend.

PRN protein is a non-ciliary outer membrane protein produced by Bordetella pertussis, as an important virulence factor, PRN protein plays an important role in bacterial infection and adhesion to the epithelial cell membrane of the host respiratory system. PRN protein is also an important protective antigen that can induce humoral and cellular immune responses in mice. PRN protein has shown a good protection rate in the respiratory tract attack animal model test of Bordetella pertussis[20]. According to the difference in the structure of this region and amino acids at specific positions, the prn gene is divided into prn1-12 subtypes[21, 24]. Studies in some countries have confirmed that prn genotypes of strains isolated before or in the early stages of inoculation and pertussis vaccine strain are mainly prn1 genotypes. Since the 1980s, prn2 subtypes have appeared in some European countries. By 2000 s, prn2 or prn3 had been the dominant genotypes of the isolates in the Netherlands, Finland, France, the United States, and other countries[25,26]. Bioinformatics analyses of the amino acid secondary structure deduced from the prn gene sequence of strains isolated in different ages show that the prn genotype structure of the strains isolated after the 2000s changed to a certain extent, and the hydrophilic region appeared, which led to the change of its immunogenicity. However, the previously isolated prn1》prn7区 prn10 and prn11 are a completely hydrophobic region in this region 1[27]. In this study, $62 \%$ of the isolates belonged to $p r n 2$ and $2 \%$ belonged to $p r n 3$, which was consistent with the evolution trend of $p r n$ genes in the world.

Clinical trials in some countries have reported that there is a good correlation between the anti-Fim protein antibody titer in the pertussis vaccine and the immune response in mice and the immune vaccine protection in the children. WHO guidelines for pertussis vaccine recommend that strains expressing of type 2 and 3 Fim protein antigens should be selected as the production strain in the pertussis vaccine production. As reported, these two fimbriae proteins also have certain immunogenicity and have been used as components of acellular pertussis vaccine by some vaccine manufacturers. In this study, the isolates contained the same fim2-1 and fim3-1 genes as the vaccine strain, indicating that these two antigen-related genes are relatively conservative.

In this study, only $16.7 \%$ of the children had completed the full vaccination of pertussis vaccine, and about $71.4 \%$ of positive patients had not completed the basic process of APV vaccination at the time of onset, mainly because these children were relatively young at the age of onset and had not yet reached the planned immunization. The current pertussis immunization targets in China do not include pregnant women, resulting in infants receiving little pertussis antibody from the mother, and the first dose of 
vaccination for the child is 3 months old, leading to a longer unprotected window a long-unprotected window for infants of younger months. These may be important reasons for the high incidence of pertussis in China. Another possible reason is that the effectiveness of the pertussis vaccine declines rapidly over time[28,29]. Studies have shown that the pertussis vaccine is highly effective within three

years of vaccination, but then the immunity gradually weakens, with little protection after seven years[30, 31].

Since the APV vaccine was widely used worldwide at the late 1990s, in addition to FHA, other components including PT, PRN, Fim2, and Fim3 showed different degrees of antigenic gene polymorphisms between vaccine strains and isolated epidemic strains, resulting in a significant change in the population of Bordetella pertussis. That explains why strains isolated in regions or countries with low vaccination coverage are similar to those circulating before the introduction of the vaccine. Therefore, molecular monitoring of epidemic strains is particularly important. This study recommends that bacterial typing methods (such as the MLVA method) should be used to add information on pertussis epidemic strains in different countries or regions to the established database, and to gradually establish a pertussis epidemic surveillance system that can be shared by global public health departments and researchers.

\section{Conclusions}

This study explained the resurgence of whooping cough from certain angles, including immunization strategy, vaccination time and genome variation of strains, which is beneficial to prevent pertussis infections.

\section{Declarations}

\section{Availability of data and materials}

The data that support the findings of this study have been deposited into CNGB Sequence Archive (CNSA) [32] of China National GeneBank DataBase (CNGBdb) [33] with accession number CNP0001528. The datasets generated for this study are available on request to the corresponding author.

\section{Ethics approval and consent to participate}

Bordetella pertussis isolates from the Shenzhen Center for Disease Control and Prevention were deidentified and anonymized to protect patient privacy and confidentiality; therefore, ethical clearance was not required.

\section{Consent for publication}

Not applicable.

\section{Authors' contributions}


Conceived and designed the experiments: SW, XS, QH; performed the experiments: SW, HC; contributed analysis: SW, QH, CY, HZ, YZ, MJ; wrote the paper: SW, QH, XS, YH

\section{Funding}

This research was supported by the National Major Science and Technology Projects of China (No. 2017ZX10303406, No. 2018ZX10714002-003-010), Sanming Project of Medicine in Shenzhen (NO.SZSM201811071), National Natural Science Foundation of China (No.81773436, No.81673174).

\section{Acknowledgements}

The authors would like to express their deepest gratitude to the Laboratory of Medicine of Shenzhen Children's Hospital and the National Institute for Communicable Disease Control and Prevention (ICDC) of China CDC.

\section{Competing interests}

The authors declare that the research was conducted in the absence of any commercial or financial relationships that could be construed as a potential conflict of interest.

\section{References}

1. Esposito S, Stefanelli P, Fry NK, Fedele G, He Q, Paterson P, et al. Pertussis Prevention: Reasons for Resurgence, and Differences in the Current Acellular Pertussis Vaccines. Frontiers in immunology. 2019;10:1344.

2. Zimmerman LI, Papin JF, Warfel J, Wolf RF, Kosanke SD, Merkel TJ. Histopathology of Bordetella pertussis in the Baboon Model. Infection and immunity. 2018;86(11).

3. Schellekens J, von König CH, Gardner P. Pertussis sources of infection and routes of transmission in the vaccination era. The Pediatric infectious disease journal. 2005;24(5 Suppl):S19-24.

4. Kuchar E, Karlikowska-Skwarnik M, Han S, Nitsch-Osuch A. Pertussis: History of the Disease and Current Prevention Failure. Advances in experimental medicine and biology. 2016;934:77-82.

5. Berkovic SF, Harkin L, McMahon JM, Pelekanos JT, Zuberi SM, Wirrell EC, et al. De-novo mutations of the sodium channel gene SCN1A in alleged vaccine encephalopathy: a retrospective study. The Lancet Neurology. 2006;5(6):488-92.

6. Reyes IS, Hsieh DT, Laux LC, Wilfong AA. Alleged cases of vaccine encephalopathy rediagnosed years later as Dravet syndrome. Pediatrics. 2011;128(3):e699-702.

7. Domenech de Cellès M, Magpantay FM, King AA, Rohani P. The pertussis enigma: reconciling epidemiology, immunology and evolution. Proc Biol Sci. 2016;283(1822).

8. Jackson DW, Rohani P. Perplexities of pertussis: recent global epidemiological trends and their potential causes. Epidemiol Infect. 2014;142(4):672-84. 
9. Robbins JB, Schneerson R, Trollfors B. Pertussis in developed countries. The Lancet. 2002;360(9334):657-8.

10. Mooi FR, van Loo IH, van Gent M, He Q, Bart MJ, Heuvelman KJ, et al. Bordetella pertussis strains with increased toxin production associated with pertussis resurgence. Emerg Infect Dis. 2009;15(8):1206-13.

11. Pertussis epidemic-Washington, 2012. MMWR Morbidity and mortality weekly report. 2012;61(28):517-22.

12. Xu Y, Tan Y, Asokanathan C, Zhang S, Xing D, Wang J. Characterization of co-purified acellular pertussis vaccines. Human vaccines \& immunotherapeutics. 2015;11(2):421-7.

13. Clarke MF, Rasiah K, Copland J, Watson M, Koehler AP, Dowling K, et al. The pertussis epidemic: informing strategies for prevention of severe disease. Epidemiol Infect. 2013;141(3):463-71.

14. Safarchi A, Octavia S, Wu SZ, Kaur S, Sintchenko V, Gilbert GL, et al. Genomic dissection of Australian Bordetella pertussis isolates from the 2008-2012 epidemic. The Journal of infection. 2016;72(4):468-77.

15. Hozbor D, Ulloa-Gutierrez R, Marino C, Wirsing von König CH, Tan T, Forsyth K. Pertussis in Latin America: Recent epidemiological data presented at the 2017 Global Pertussis Initiative meeting. Vaccine. 2019;37(36):5414-21.

16. Xu Z, Wang Z, Luan Y, Li Y, Liu X, Peng X, et al. Genomic epidemiology of erythromycin-resistant Bordetella pertussis in China. Emerging microbes \& infections. 2019;8(1):461-70.

17. Yu W, Lee LA, Liu Y, Scherpbier RW, Wen N, Zhang G, et al. Vaccine-preventable disease control in the People's Republic of China: 1949-2016. Vaccine. 2018;36(52):8131-7.

18. Bart MJ, Harris SR, Advani A, Arakawa Y, Bottero D, Bouchez V, et al. Global population structure and evolution of Bordetella pertussis and their relationship with vaccination. mBio. 2014;5(2):e01074.

19. Martin SW, Pawloski L, Williams M, Weening K, DeBolt C, Qin X, et al. Pertactin-negative Bordetella pertussis strains: evidence for a possible selective advantage. Clinical infectious diseases : an official publication of the Infectious Diseases Society of America. 2015;60(2):223-7.

20. Smith AM, Guzmán CA, Walker MJ. The virulence factors of Bordetella pertussis. a matter of control. FEMS microbiology reviews. 2001;25(3):309-33.

21. Mooi FR, van Oirschot $H$, Heuvelman K, van der Heide HG, Gaastra W, Willems RJ. Polymorphism in the Bordetella pertussis virulence factors P.69/pertactin and pertussis toxin in The Netherlands: temporal trends and evidence for vaccine-driven evolution. Infection and immunity. 1998;66(2):670-5.

22. Gzyl A, Augustynowicz E, Gniadek G, Rabczenko D, Dulny G, Slusarczyk J. Sequence variation in pertussis S1 subunit toxin and pertussis genes in Bordetella pertussis strains used for the whole-cell pertussis vaccine produced in Poland since 1960: efficiency of the DTwP vaccine-induced immunity against currently circulating B. pertussis isolates. Vaccine. 2004;22(17-18):2122-8.

23. Parkhill J, Sebaihia M, Preston A, Murphy LD, Thomson N, Harris DE, et al. Comparative analysis of the genome sequences of Bordetella pertussis, Bordetella parapertussis and Bordetella bronchiseptica. Nature genetics. 2003;35(1):32-40. 
24. Kallonen T, He Q. Bordetella pertussis strain variation and evolution postvaccination. Expert review of vaccines. 2009;8(7):863-75.

25. Preston NW, Carter EJ. Serotype specificity of vaccine-induced immunity to pertussis. Communicable disease report CDR review. 1992;2(13):R155-6.

26. Olin P, Rasmussen F, Gustafsson L, Hallander HO, Heijbel H. Randomised controlled trial of twocomponent, three-component, and five-component acellular pertussis vaccines compared with wholecell pertussis vaccine. Ad Hoc Group for the Study of Pertussis Vaccines. Lancet (London, England). 1997;350(9091):1569-77.

27. Packard ER, Parton R, Coote JG, Fry NK. Sequence variation and conservation in virulence-related genes of Bordetella pertussis isolates from the UK. Journal of medical microbiology. 2004;53(Pt 5):355-65.

28. Warfel JM, Edwards KM. Pertussis vaccines and the challenge of inducing durable immunity. Current opinion in immunology. 2015;35:48-54.

29. McGirr A, Fisman DN. Duration of pertussis immunity after DTaP immunization: a meta-analysis. Pediatrics. 2015;135(2):331-43.

30. Schwartz KL, Kwong JC, Deeks SL, Campitelli MA, Jamieson FB, Marchand-Austin A, et al. Effectiveness of pertussis vaccination and duration of immunity. CMAJ : Canadian Medical Association journal = journal de l'Association medicale canadienne. 2016;188(16):E399-e406.

31. Pool V, Tomovici A, Johnson DR, Greenberg DP, Decker MD. Humoral immunity 10 years after booster immunization with an adolescent and adult formulation combined tetanus, diphtheria, and 5component acellular pertussis vaccine in the USA. Vaccine. 2018;36(17):2282-7.

32. Guo XQ, Chen FZ, Gao F, et al. CNSA: a data repository for archiving omics data. Database (Oxford). 2020;2020:baaa055. doi:10.1093/database/baaa055.

33. Chen FZ, You LJ, Yang F, et al. CNGBdb: China National GeneBank DataBase. Hereditas. 2020;42(08):799-809. doi:10.16288/j.yczz.20-080.

\section{Tables}

\section{Table 1. Epidemiological characteristics and vaccination status of children}




\begin{tabular}{|c|c|c|c|c|c|c|c|}
\hline \multirow[t]{2}{*}{ Number of immunizations } & \multirow[t]{2}{*}{ Number of cases } & \multicolumn{2}{|c|}{ Gender } & \multicolumn{4}{|c|}{ Age (months) } \\
\hline & & Male & female & $0-3$ & $4-6$ & $7-12$ & $>12$ \\
\hline 0 & 11 & 8 & 3 & 9 & 2 & 0 & 0 \\
\hline 1 & 13 & 6 & 7 & 1 & 9 & 2 & 1 \\
\hline 2 & 6 & 3 & 3 & 1 & 2 & 2 & 1 \\
\hline 3 & 5 & 2 & 3 & 0 & 0 & 2 & 3 \\
\hline 4 & 7 & 3 & 4 & 0 & 0 & 0 & 7 \\
\hline
\end{tabular}

Table 2. Main virulence genotypes of clinical isolates in Shenzhen

\begin{tabular}{|c|c|c|}
\hline genotype & Strain number & proportion \\
\hline \multirow{2}{*}{$\begin{array}{l}\text { prn1/ptxA1/ptxP1/ptxC1/fim3- } \\
1 / f i m 2-1\end{array}$} & BG03,04,07,13,15,17,24,29,30,31,32,33,34,35,37, & \multirow[t]{2}{*}{$18 \rrbracket 36 \% \rrbracket$} \\
\hline & $45,46,47$ & \\
\hline $\begin{array}{l}\text { prn2/ptxA1/ptxP3/ptxC2/fim3- } \\
1 / f i m 2-1\end{array}$ & $\begin{array}{l}\text { BG01,02,06,08,09,10,11,12,14,16,18,19,20,21,22, } \\
23,25,26,27,28,36,38,39,40,41,42,43,44,48,49,50\end{array}$ & $31 \rrbracket 62 \% \rrbracket$ \\
\hline $\begin{array}{l}\text { prn3/ptxA1/ptxP1/ptxC1/fim3- } \\
1 / f i m 2-1\end{array}$ & BG05 & $1 \rrbracket 2 \% \rrbracket$ \\
\hline $\begin{array}{l}\text { prn1/ptxA2/ptxP1/ptxC1/fim3- } \\
1 / f i m 2-1\end{array}$ & 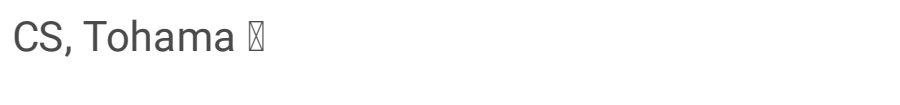 & $0(0 \%)$ \\
\hline
\end{tabular}

Figures 


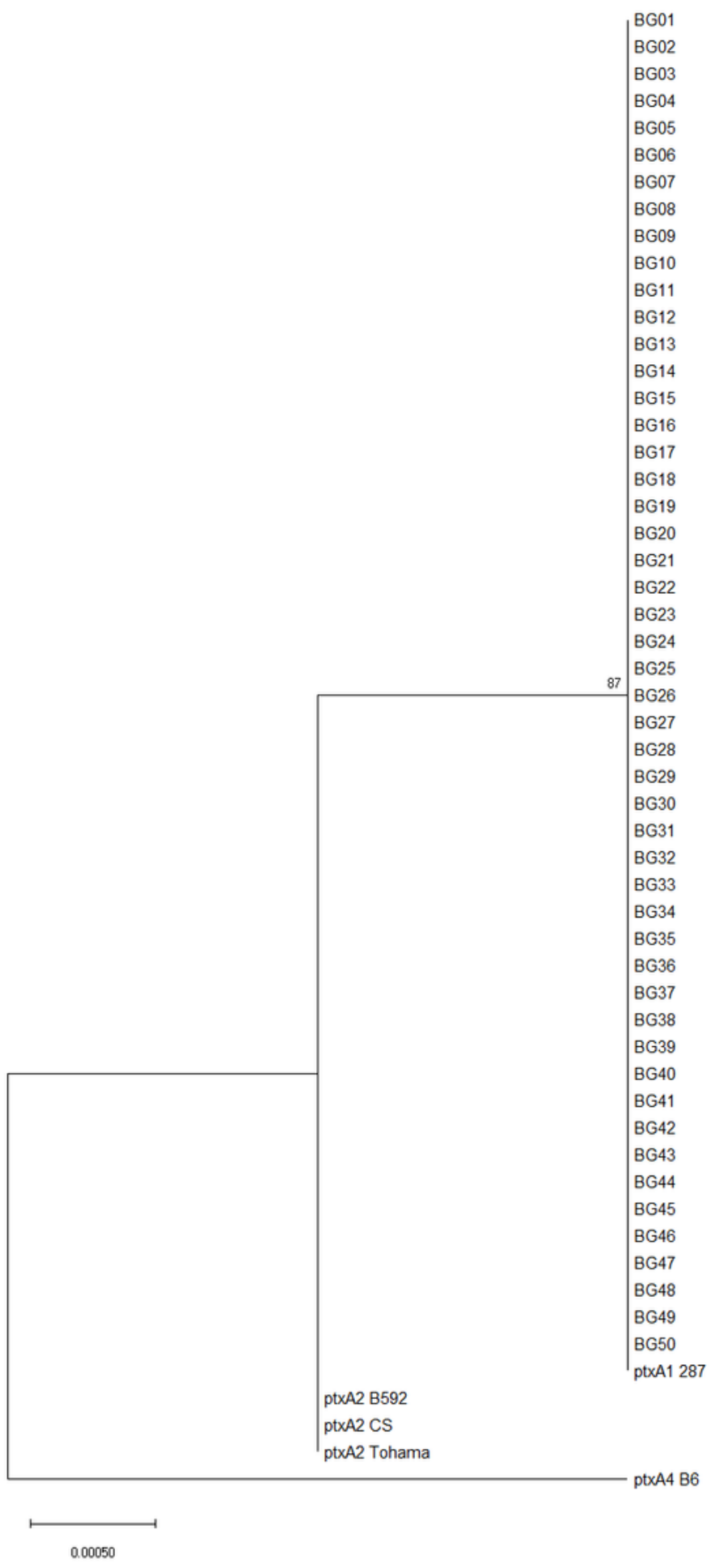

Figure 1

Genotype analysis of ptxA isolates 


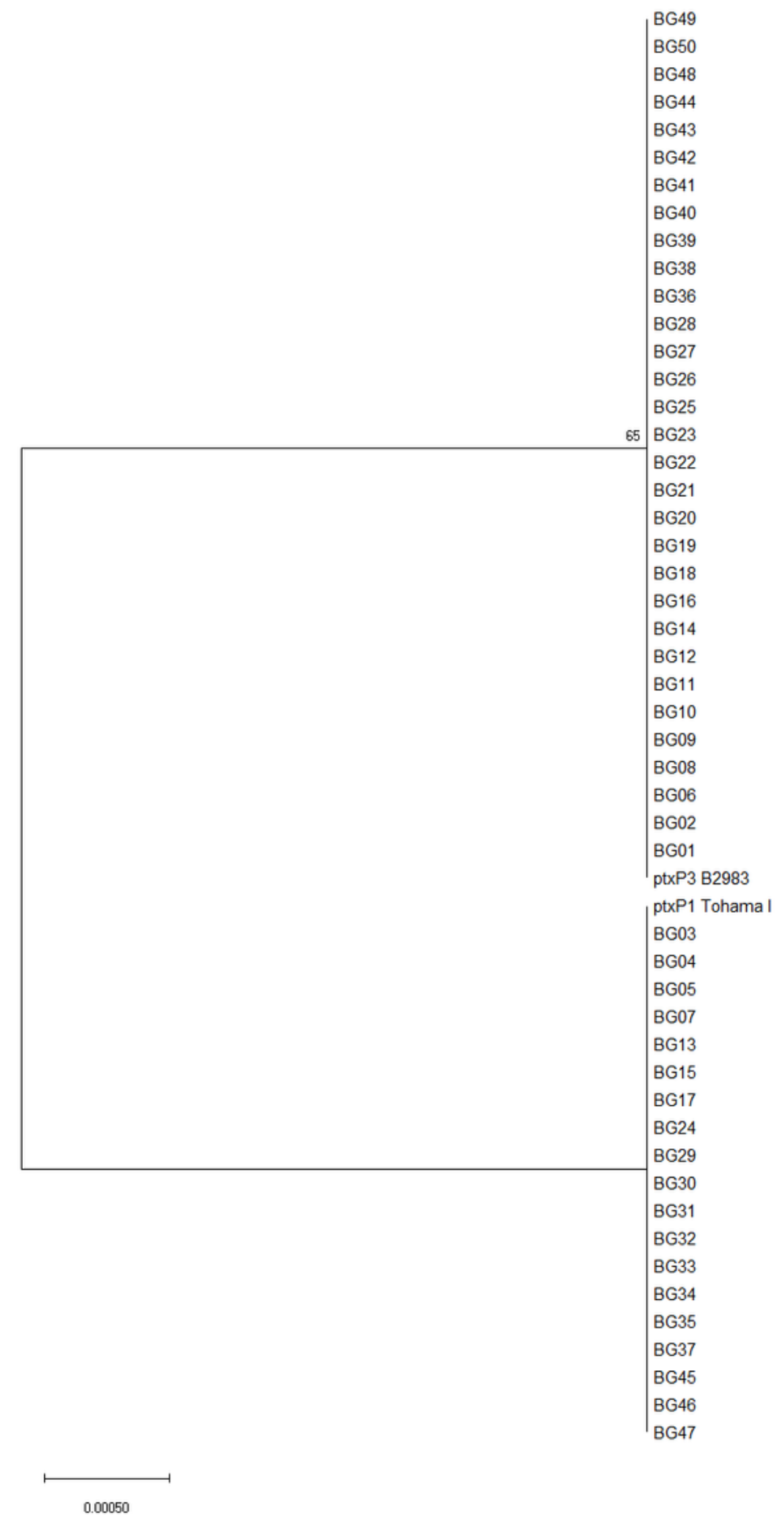

Figure 2

Genotype analysis of ptxP isolates 


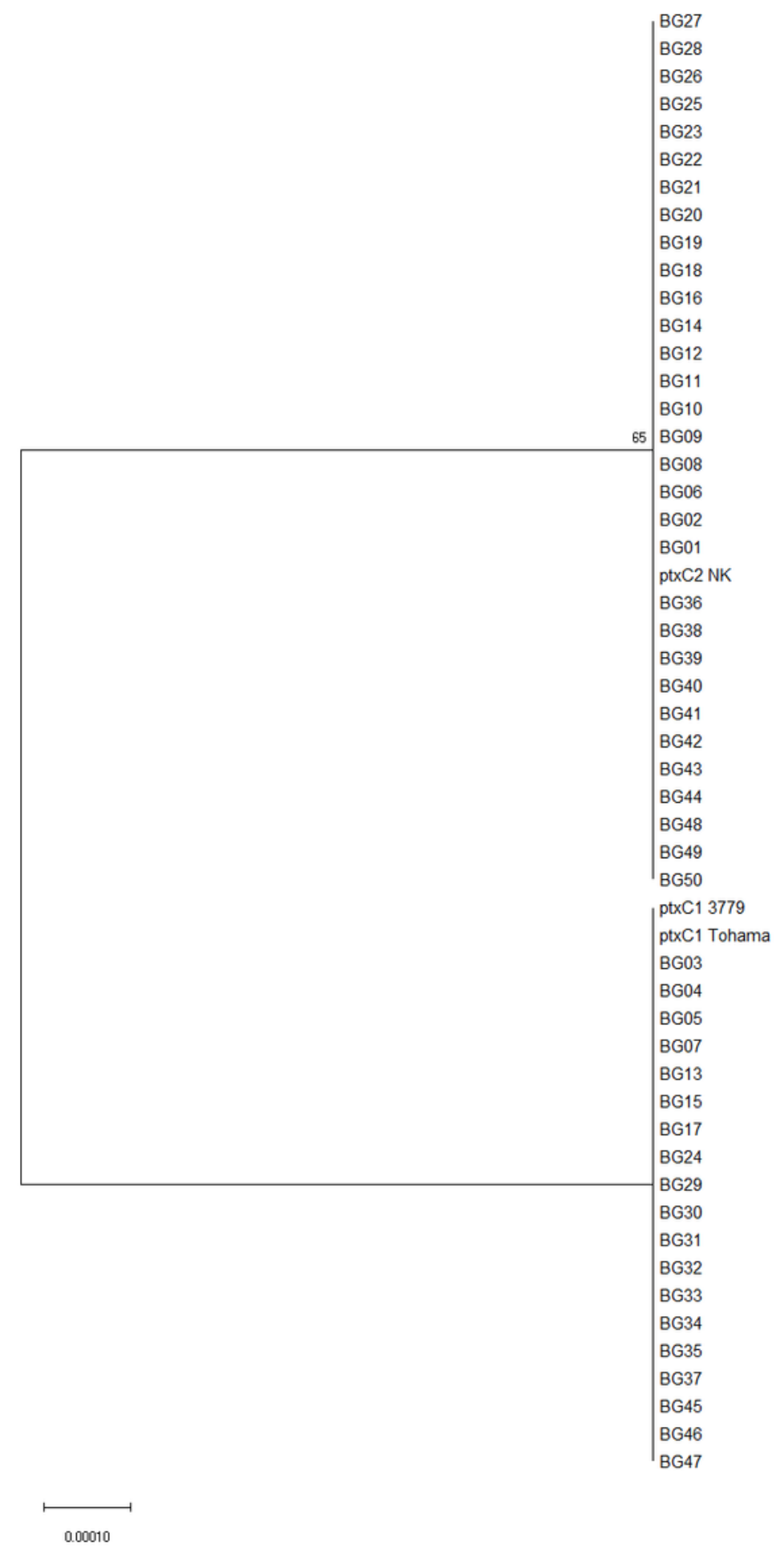

Figure 3

Genotype analysis of ptxC isolates 


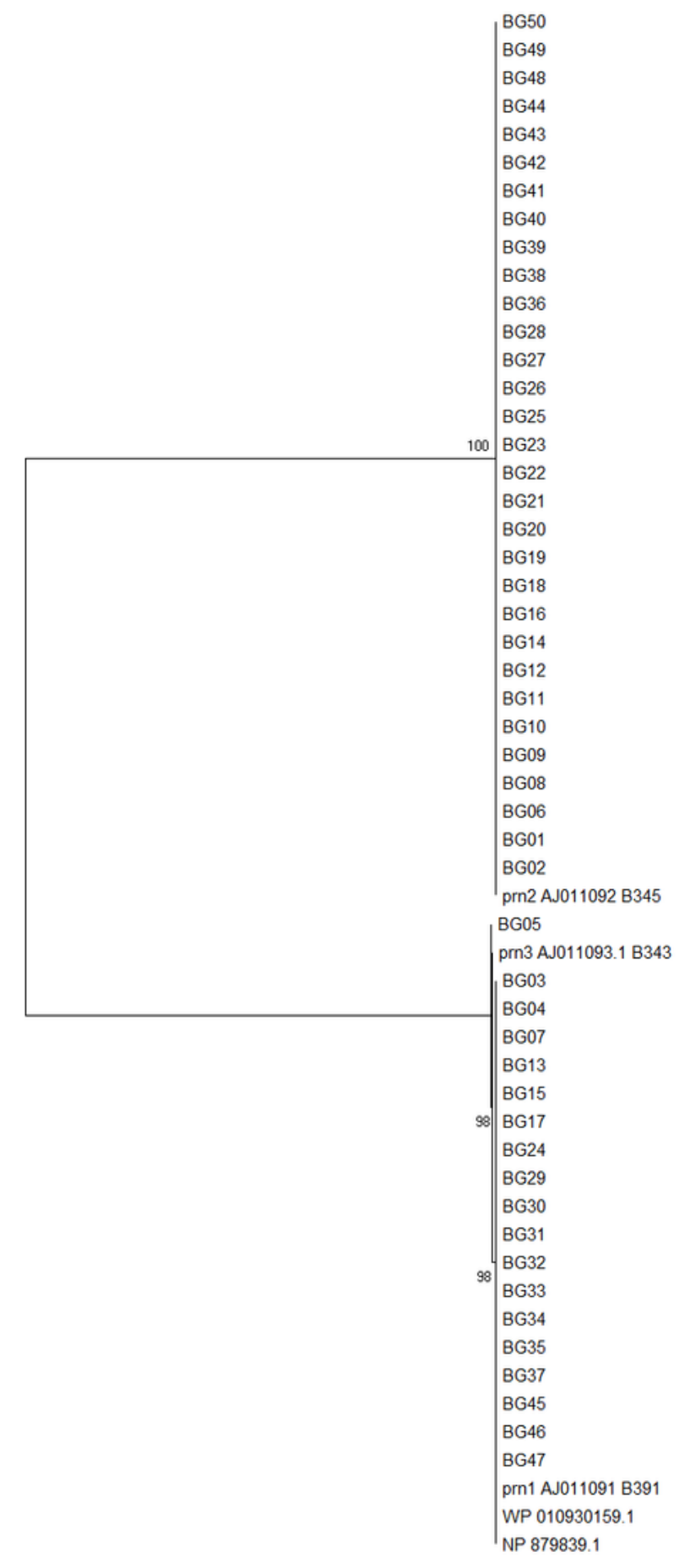

0.20

Figure 4

Genotype analysis of prn isolates 


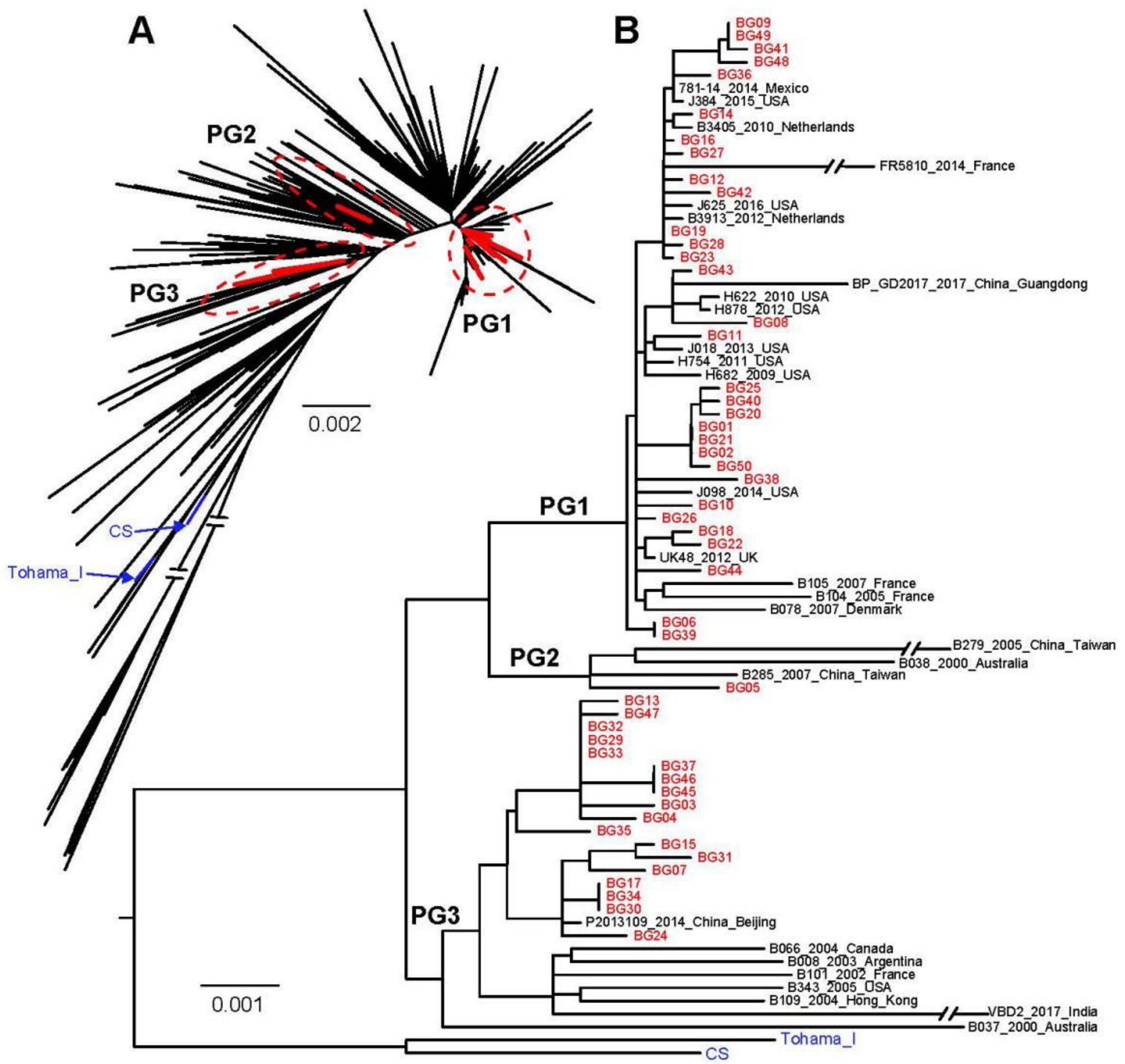

Figure 5

The phylogenetic tree of Shenzhen strains and international strains. A: Maximum likelihood phylogenetic tree of 50 Shenzhen strains and 842 international strains. B: Maximum likelihood phylogeny tree of Shenzhen strain, phylogenetic branch PG1-PG3 strain and reference strain. Red and blue represented the Shenzhen strain and two reference strains, respectively. For better visualization, the longer phylogenetic branches were artificially shortened (double slashes). 